Research, Society and Development, v. 9, n. 11, e1609119715, 2020

(CC BY 4.0) | ISSN 2525-3409 | DOI: http://dx.doi.org/10.33448/rsd-v9i11.9715

Perfil sociodemográfico e epidemiológico de pacientes renais crônicos em terapia hemodialítica

Sociodemographic and epidemiological profile of chronic kidney patients in hemodialytic therapy

Perfil sociodemográfico y epidemiológico de los pacientes renales crónicos en hemodiálises

Recebido: 28/10/2020 | Revisado: 02/11/2020 | Aceito: 04/11/2020 | Publicado: 08/11/2020

Edildete Sene Pacheco

ORCID: https://orcid.org/0000-0002-5329-8037

Universidade Federal do Piauí, Brasil

E-mail: edildete_sene@hotmail.com

Isabela Santana Macêdo

ORCID: https://orcid.org/0000-0002-3705-8930

Faculdade Única de Ipatinga, Brasil

E-mail: belamacedo39@gmail.com

Gabriela Santana Macêdo

ORCID: https://orcid.org/0000-0002-3136-4814

Faculdade Sudoeste, Brasil

E-mail: gabrielamacedo060@gmail.com

Aclênia Maria Nascimento Ribeiro

ORCID: https://orcid.org/0000-0002-5582-9663

Universidade Federal do Piaú, Brasil

E-mail: aclennya@hotmail.com

Mariana Pereira Barbosa Silva

ORCID: https://orcid.org/0000-0003-0852-8099

Universidade Estadual do Piauí, Brasil

E-mail: marianapbsilvaa@gmail.com

Gabriela Oliveira Parentes da Costa

ORCID: https://orcid.org/0000-0001-9473-8986

Instituto Federal do Maranhão, Brasil

E-mail: gabiparents@ @otmail.com 


\section{Francinalda Pinheiro Santos}

ORCID: https://orcid.org/0000-0003-1222-7854

Instituto de Ensino Superior Múltiplo, Brasil

E-mail: franfilho2@hotmail.com

Elton Filipe Pinheiro de Oliveira

ORCID: https://orcid.org/0000-0003-0930-859X

Universidade Federal do Piauí, Brasil

E-mail: helton-oliver@hotmail.com

Felipe Nascimento Vidal

ORCID: https://orcid.org/0000-0001-6506-7706

Hospital Universitário da Universidade Federal do Piauí, Brasil

E-mail: felipenvidal@yahool.com.br

Evellyn Stefanne Bastos Marques

ORCID: https://orcid.org/0000-0002-8325-6188

Universidade Federal do Piauí, Brasil

E-mail: evellynbastos15@gmail.com

Agna Roberta Rodrigues de Sousa

ORCID: https://orcid.org/0000-0002-7923-2335

Instituto Brasileiro de Terapia Intensiva, Brasil

E-mail: agnaenf@hotmail.com

\section{Resumo}

Objetivo: Descrever o perfil sociodemográfico e epidemiológico dos pacientes com doença renal crônica em terapêutica hemodialítica. Metodologia: Trata-se de uma pesquisa exploratória, descritiva e quantitativa, realizada com 85 pacientes em terapia hemodialítica de uma clínica de nefrologia localizada em Floriano/PI. A coleta de dados foi realizada no mês de maio de 2017 após a aprovação do Comitê de Ética em Pesquisa sob o número do parecer 2.193.692, utilizando um roteiro de entrevista com variáveis divididas em dois grupos: sociodemográfico e epidemiológico, elaborado pelos pesquisadores. Resultados: Foi observado que a maioria desses pacientes constitui-se de indivíduos do sexo masculino (65\%), com idade compreendida entre 56 a 70 anos (36\%), estado civil casado (60\%), aposentado (35\%), residentes no estado do Piauí (83\%), com ensino fundamental incompleto $(31,7 \%)$ e declarados católicos $(51 \%)$. Quanto ao perfil epidemiológico, constatou-se como causa principal da lesão renal crônica, a hipertensão arterial sistêmica (63\%), seguida de 
diabetes mellitus (20,5\%). Observou-se ainda que a maioria dos entrevistados realizam diálise há um longo período de tempo $(25,7 \%)$ e que o tipo de acesso vascular utilizado para a realização da diálise é a fistula arteriovenosa (82,8\%). Conclusão: Em vista disso, conclui-se que a identificação e análise do perfil dos pacientes podem subsidiar os profissionais de saúde atuantes na área clínica, facilitando o manejo das atividades desenvolvidas em campo.

Palavras-chave: Insuficiência renal crônica; Diálise renal; Perfil de saúde.

\begin{abstract}
Objective: To describe the sociodemographic and epidemiological profile of patients with chronic kidney disease undergoing hemodialysis. Methodology: This is an exploratory, descriptive and quantitative research carried out with 85 patients undergoing hemodialysis at a nephrology clinic located in Floriano/PI. Data collection was carried out in May 2017 after approval by the Research Ethics Committee under the opinion number 2.193.692, using an interview script with variables divided into two groups: sociodemographic and epidemiological, prepared by the researchers. Results: It was observed that the majority of these patients are male (65\%), aged between 56 to 70 years old (36\%), married marital status $(60 \%)$, retired $(35 \%)$, residents in the state of Piauí $(83 \%)$, with incomplete elementary education $(31.7 \%)$ and declared Catholic (51\%). Regarding the epidemiological profile, systemic arterial hypertension (63\%) was found to be the main cause of chronic kidney injury, followed by diabetes mellitus (20.5\%). It was also observed that the majority of respondents have been undergoing dialysis for a long time (25.7\%) and that the type of vascular access used to perform dialysis is the arteriovenous fistula (82.8\%). Conclusion: In view of this, it is concluded that the identification and analysis of the profile of the patients can subsidize the health professionals working in the clinical area, facilitating the management of the activities developed in the field.
\end{abstract}

Keywords: Renal insufficiency chronic; Renal dialysis; Health profile.

\title{
Resumen
}

Objetivo: Describir el perfil sociodemográfico y epidemiológico de los pacientes con enfermedad renal crónica en hemodiálisis. Metodología: Se trata de una investigación exploratoria, descriptiva y cuantitativa realizada con 85 pacientes en hemodiálisis en una clínica de nefrología ubicada en Floriano / PI. La recolección de datos se realizó en mayo de 2017 luego de la aprobación del Comité de Ética en Investigación con el número 2.193.692, mediante un guión de entrevista con variables divididas en dos grupos: sociodemográficas y 
epidemiológicas, elaborado por los investigadores. Resultados: Se observó que la mayoría de estos pacientes son hombres (65\%), con edades entre 56 y 70 años (36\%), estado civil casado (60\%), jubilados (35\%), residentes en el estado de Piauí (83\%), con educación primaria incompleta $(31,7 \%)$ y declarada católica $(51 \%)$. En cuanto al perfil epidemiológico, se encontró que la hipertensión arterial sistémica (63\%) es la principal causa de daño renal crónico, seguida de la diabetes mellitus (20,5\%). También se observó que la mayoría de los encuestados lleva mucho tiempo en diálisis $(25,7 \%)$ y que el tipo de acceso vascular utilizado para realizar la diálisis es la fístula arteriovenosa (82,8\%). Conclusión: Ante esto, se concluye que la identificación y análisis del perfil de los pacientes puede subsidiar a los profesionales de la salud que laboran en el área clínica, facilitando la gestión de las actividades desarrolladas en el campo.

Palabra clave: Insufíciencia renal crónica; Diálise renal; Perfil de salud.

\section{Introdução}

Atualmente as doenças degenerativas constituem um dos grandes problemas de saúde pública, sendo responsável por taxas elevadas de morbimortalidade mundialmente. Nesse contexto, podemos incluir a doença renal crônica (DRC), que é descrita como uma problemática que acarreta prejuízo gradual e irreversível da função renal e que necessita de uma terapêutica complexa e de custos elevados (Nunes et al., 2013; Draczevski \& Teixeira, 2011).

É importante que o diagnóstico da DRC seja dado o mais precocemente possível, pois quando isso ocorre, o paciente consegue ter significativa qualidade de vida e tratamento adequado. O diagnóstico realizado precocemente também favorece a diminuição dos gastos públicos. As formas de tratamento disponíveis são: tratamento medicamentoso, diálise, hemodiálise e transplante renal. Somado a isso, é fundamental que a pessoa acometida tenha novos hábitos de vida, considerando as mudanças de hábitos alimentares e da ingesta hídrica (Moura et al., 2015; Melo et al., 2014).

Segundo Pivatto e Abreu (2010), a hemodiálise é um tratamento no qual o sangue é filtrado por meio de uma máquina que realiza essa filtração artificialmente. Nessa máquina, ocorre a circulação do sangue através de um rim artificial cheio de tubos com membranas semipermeáveis. Essa terapia é indicada para tratar a forma aguda e crônica da doença e que requer intervenção imediata, bem como para pacientes com DRC que permanecem em programa hemodialítico (Almeida et al., 2013). 
Research, Society and Development, v. 9, n. 11, e1609119715, 2020

(CC BY 4.0) | ISSN 2525-3409 | DOI: http://dx.doi.org/10.33448/rsd-v9i11.9715

É importante ressaltar que entre as terapias disponibilizadas para a DRC, a hemodiálise está presente na maioria dos casos. Assim o conhecimento do perfil epidemiológico dos pacientes renais crônicos em tratamento hemodialítico é essencial, pois contribui para a promoção da saúde a indivíduos vulneráveis ao adoecimento pela DRC e favorece o controle de doenças associadas, com consequente redução do índice de mortalidade desses pacientes e melhoria na qualidade de vida (Ortiz et al., 2014; Lopez et al., 2014).

Em vista disso, objetivou-se com este estudo descrever o perfil sociodemográfico e epidemiológico dos pacientes com doença renal crônica em terapêutica hemodialítica.

\section{Metodologia}

Trata-se de uma pesquisa exploratória e descritiva que presume um vasto conhecimento em relação aos fenômenos e problemáticas estudadas. A pesquisa exploratória, tem o objetivo primordial de descrever ou qualificar a natureza das variáveis que se deseja conhecer (Koche, 2011).

Quanto ao caráter da pesquisa, trata-se de uma pesquisa quantitativa, que segundo Pereira et al. (2018) envolve uma coleta de dados realizada por meio do uso de medições de grandezas e obtém-se por meio da metrologia, números com suas respectivas unidades, e foi realizada com 85 pacientes em terapia hemodialítica de uma clínica de nefrologia localizada em Floriano/PI.

A coleta de dados foi realizada no mês de maio de 2017 após a aprovação do Comitê de Ética em Pesquisa sob o número do parecer 2.193.692, utilizando um roteiro de entrevista com variáveis divididas em dois grupos: sociodemográfico e epidemiológico, elaborado pelos pesquisadores.

Os critérios de inclusão foram os pacientes com doença renal crônica da referida instituição, de ambos os sexos. Já os critérios de exclusão foram pacientes com a faixa etária inferior a 15 anos e que não tinham condições físicas e psicológicas para responder à entrevista.

Os dados foram elaborados em banco de dados com o auxílio do software Microsoft Excel 2010 para tabulação dos resultados, pelos procedimentos de cálculo para a obtenção de valores absolutos e percentuais, apresentados em tabelas. 


\section{Resultados e Discussão}

A pesquisa foi realizada com uma amostra de 85 pacientes com diagnóstico de DRC que realizam tratamento hemodialítico, investigando o perfil socioedemográfico, destacando o gênero, idade, local de moradia, escolaridade, estado civil, profissão e religião, observados na Tabela 1.

Tabela 1 - Perfil sociedemográfico dos pacientes com doença renal crônica, Floriano - 2017.

\begin{tabular}{|c|c|c|}
\hline VARIÁVEIS & $\mathbf{N}$ & $\%$ \\
\hline \multicolumn{3}{|l|}{ Gênero } \\
\hline Feminino & 30 & 35 \\
\hline Masculino & 55 & 65 \\
\hline \multicolumn{3}{|l|}{ Idade } \\
\hline 15 a 24 anos & 10 & 12 \\
\hline 25 a 39 anos & 25 & 29 \\
\hline 40 a 55 anos & 17 & 20 \\
\hline 56 a 70 anos & 31 & 36 \\
\hline 71 a 85 anos & 2 & 3 \\
\hline \multicolumn{3}{|l|}{ Local de moradia } \\
\hline Piauí & 71 & 83 \\
\hline Maranhão & 14 & 17 \\
\hline \multicolumn{3}{|l|}{ Escolaridade } \\
\hline Não alfabetizado & 25 & 29,4 \\
\hline Alfabetizado & 5 & 5,8 \\
\hline Ensino fundamental incompleto & 32 & 37,6 \\
\hline Ensino fundamental completo & 5 & 5,8 \\
\hline Ensino médio completo & 14 & 16,4 \\
\hline Ensino médio incompleto & 2 & 2,3 \\
\hline Ensino superior incompleto & 2 & 2,3 \\
\hline Ensino superior completo & 0 & 0 \\
\hline \multicolumn{3}{|l|}{ Estado civil } \\
\hline Solteiro & 22 & 25,8 \\
\hline Casado & 51 & 60 \\
\hline
\end{tabular}


Viúvo

Divorciado/separado

União estável

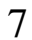

5

0
8,5

5,7

0

\section{Profissão}

Serviço braçal

Dona de casa

Estudante

Aposentado

Desempregado

Afastado do serviço

\section{3}

23

2

30

21

6
4

27

2

35

25

7

\begin{tabular}{lcc} 
Religião & 43 & 51 \\
Católica & 2 & 2 \\
Espírita & 33 & 39 \\
Evangélica & 7 & 8 \\
Outras & 7 \\
\hline
\end{tabular}

Fonte: Autores.

Pode-se observar que o gênero predominante é sexo masculino com $65 \%$ e o feminino com $35 \%$, porém, nota-se que DRC atinge tanto homens quanto mulheres indistintamente.

No que se refere à faixa etária o grupo estudado foi caracterizado dentro 15 a 85 anos no período da pesquisa. Desses, a faixa etária que prevaleceu encontra-se entre 56 a 70 anos (36\%), seguido pelo grupo de 25 a 39 anos (29\%).

Para Alves et al. (2007), apesar do processo de envelhecimento não está necessariamente pertencente a doenças e incapacidades, as doenças crônico-degenerativas são frequentemente encontradas entre os idosos. Assim, a tendência atual é termos um número crescente de indivíduos idosos que, apesar de viverem mais, apresentam maiores condições crônicos.

O número de indivíduos com idade mais avançada em tratamento hemodialítico é devido às doenças associadas como o diabetes mellitus tipo 2 e a hipertensão arterial (Cordeiro et al. 2009).

Por outro lado, faixa etária entre 18 e 59 anos, considerada economicamente ativa na sociedade, possui um comprometimento da capacidade funcional devido ao tratamento de hemodiálise, dificultando a atividade laboral, restrição hídrica e alimentar, fatores esses que 
estão incluídos dentro das condições que causam estresse em alguns clientes com DRC, tornando-se fatores que dificultam a realização do tratamento hemodialítico.

No presente estudo, quanto ao local de moradia, foi observado que a maior parte dos pacientes mora no estado do Piauí, com $83 \%$, porém grande parte dos pacientes possui dificuldades para chegar ao local do tratamento hemodialitico.

Segundo Madeiro et al. (2010), o deslocamento dos pacientes para o tratamento, por estradas não pavimentadas, proporciona, muitas vezes, uma viagem cansativa à cidade onde se encontra a Unidade de Nefrologia.

A adesão ao tratamento de hemodiálise é considerada bastante desgastante por serem realizados três dias por semana, durante quatro horas por dia (Bastos \& Kirsztajn, 2011), necessitando de transporte. Sabe-se que as atuais condições das estradas brasileiras são precárias, sendo um fator de estresse para os pacientes renais crônicos, dificultando a realização do tratamento hemodialítico.

Esse estudo revelou um baixo nível de escolaridade entre participantes, 31,7\% com ensino fundamental incompleto, seguido de 28,5\% não alfabetizado. Esse fato chama atenção, pois o baixo nível de escolaridade influencia diretamente no tratamento hemodialítico. A escolaridade constitui-se um dos principais fatores relacionados à qualidade de vida em diferentes grupos populacionais.

De acordo com Andrade et al. (2014), a baixa escolaridade, comum às diferentes regiões do Brasil, pode representar dificuldades no entendimento das orientações realizadas no seguimento do tratamento hemodialítico.

Os pacientes que passaram a fazer hemodiálise, em alguns casos possuem a falta de entendimento ou conhecimento relacionado ao tratamento, o que gera dificuldade de adesão. A baixa escolaridade é o fator que comprova que a falta de conhecimento e informação interfere diretamente na qualidade de vida dos pacientes (Maldaner et al., 2008).

Pode-se observar que o estado civil predominante é o casado com $60 \%$, em seguida observa-se o solteiro com $25,8 \%$, viúvo $8,5 \%$, divorciado/separado com 5,7\%. Esse fato pode ser considerado como um ponto positivo, pois o acompanhante pode dar suporte ao indivíduo no diagnóstico da doença e durante o tratamento, para que não se sinta sozinho nessa batalha, possuindo assim um apoio familiar. O fato de residir com a família ou ter alguém para acompanhá-lo pode contribuir para melhorar o suporte social, assumindo função protetora, relacionado às complicações em decorrência da DRC, do tratamento hemodialítico e das comorbidades (Madeiro et al., 2010). 
Research, Society and Development, v. 9, n. 11, e1609119715, 2020

(CC BY 4.0) | ISSN 2525-3409 | DOI: http://dx.doi.org/10.33448/rsd-v9i11.9715

Em relação à ocupação, pode-se observar que é predominante os aposentados com $35 \%$, seguido por $27 \%$ das donas de casa, $25 \%$ desempregados, $7 \%$ afastados do serviço, $4 \%$ desempenhando algum serviço braçal e $2 \%$ estudantes.

Para Bertolin (2011), as pessoas em tratamento hemodialítico enfrentam dificuldades para trabalhar em decorrência do comprometimento físico, tempo designado à hemodiálise e dificuldades de contratação após início dessa terapia.

O desemprego ou a falta de possibilidade de trabalhar provoca diminuição da autoestima e esses indivíduos passam a ser dependentes financeiramente e a necessitar, na maioria das vezes, do auxílio de um familiar ou pessoa próxima (Kirchner et al., 2011).

Em muitos casos, há a inversão de papéis dentro da família, principalmente diante das necessidades financeiras, cabendo ao cônjuge saudável ir trabalhar fora de casa, enquanto que o paciente renal responsabiliza-se por algumas atividades domésticas (Costa et al., 2014).

No que se refere à religião foi observado um percentual maior de católicos com 51\%, seguida dos evangélicos com 39\%, outras religiões com $8 \%$, e espiritas com 2\%. Para Souza Júnior et al. (2015), os pacientes com doença renal crônica devem se adaptar não somente à patologia e ao seu tratamento, mas também aos numerosos problemas fisiológicos, psicossociais e espirituais que advém dessa condição. Nesse contexto desfavorável, muitos se apegam à fé e à religião como forma de encontrar apoio e alívio para seu sofrimento.

Segundo Valcantti et al. (2012), promover e possibilitar a utilização da religião/espiritualidade no processo de enfrentamento da doença, previne atitudes de pessimismo e desânimo frente à convivência com a doença e, consequentemente, uma melhora no estado geral de saúde.

Observa-se que a religião e espiritualidade têm significativa relevância no tratamento da doença renal crônica, seja para os pacientes, seja para os familiares e amigos que convivem com o sofrimento crônico causado pela doença e suas consequências (Souza Júnior et al., 2015).

Para o conhecimento de dados epidemiológico da amostra, foram destacadas as principais causas de lesão renal crônica, tempo de diálise e tipo de acesso vascular, conforme a Tabela 2. 
Tabela 2 - Perfil epidemiológico dos pacientes com doença renal crônica, Floriano - 2017.

\begin{tabular}{lcc}
\hline \multicolumn{1}{c}{ VARIÁVEIS } & N & \% \\
\hline Causa da lesão renal crônica & 8 & 10 \\
Desconhecida & 18 & 20,5 \\
Diabetes Mellitus & 54 & 63 \\
Hipertensão Arterial sistêmica & 3 & 3,5 \\
Doenças dos rins poliscísticos & 2 & 3 \\
Glomerulonefrite & & \\
Tempo de diálise & 7 & 8,5 \\
$<6$ meses & 22 & 25,7 \\
$\geq 1$ ano e $<2$ anos & 15 & 17,3 \\
$\geq 2$ anos e $<3$ anos & 14 & 17,1 \\
$\geq 4$ anos e $<5$ anos & 22 & 25,7 \\
$\geq 6$ anos e $<10$ anos & 5 & 5,7 \\
$\geq 10$ anos & 10 & 82,8 \\
Tipo de acesso vascular & & 0 \\
Fistula arteriovenosa & 70 & 11,4 \\
Prótese & 0 & \\
Cateter Central Duplo & 5 & \\
Lúmen & & \\
Cateter Central Permcath & & \\
\hline & & \\
\hline
\end{tabular}

Fonte: Autores.

A Tabela 2 expõe as principais causas de lesão renal crônica relatada pelos pacientes da unidade de tratamento onde foi realizada a pesquisa. Demostrou que $63 \%$ dos pacientes possuem hipertensão arterial, seguido por diabetes mellitus com 20,5\%, causa desconhecida atingindo $10 \%$, doenças dos rins poliscísticos com 3,5\% e sendo a menor causa de IRC, glomerulonefrite com $3 \%$.

Observando os dados apresentados, acredita-se que grande parte dos pacientes relatam ter uma doença, mais que necessariamente não significa dizer que foi a causa para DRC. De maneira geral, os pacientes não questionam a sua doença nem tão pouco percebe o motivo de seu adoecimento.

Segundo Bortolotto (2008), a hipertensão arterial e função renal estão intimamente relacionadas, podendo a hipertensão ser tanto a causa como a consequência de uma doença 
renal. A hipertensão arterial está presente na maioria das doenças renais. A prevalência de hipertensão, determinada por ocasião da detecção da doença renal, aumenta progressivamente à medida que a função renal vai deteriorando, de forma que na fase terminal ou dialítica de DRC, a quase totalidade dos nefropatias é hipertensa.

Para Vieira Júnior \& Suassuna (2013), o acometimento renal pelo diabetes corresponde por $40 \%$ dos casos incidentes de diálise nos Estados Unidos, e no Brasil rivaliza com a hipertensão, acometendo $30 \%$ dos pacientes que iniciam tratamento dialítico. Estes pacientes sofrem um risco adicional de morte de $20 \%$ no primeiro ano de diálise, principalmente por causas cardiovasculares. Em outras palavras, cerca de $30 \%$ dos pacientes com diabetes desenvolvem nefropatia diabética, especialmente em associação com hipertensão.

De acordo com o tempo de diálise, $25,7 \%$ dos pacientes fazem a diálise $\geq 1$ ano e $<2$ anos e $\geq 6$ anos e $<10$ anos, seguido por $\geq 2$ anos e $<3$ anos com 17,3\%, $\geq 4$ anos e $<5$ anos com $17,1 \%,<6$ meses atingindo $10 \%$ e sendo a menor tempo $\geq 10$ anos com $5,7 \%$.

Observou-se que o número de pacientes realizando o tratamento entre 6 a 10 anos tem o valor igual ao de 1 a 2 anos. Trazendo a perspectiva que aumentaram o número de pacientes que realizam diálise por longos períodos de tempo.

Para Silva et al. (2016), com as repercussões positivas das melhorias diagnósticas, terapêuticas e da qualidade global do programa dialítico oferecido atualmente favorece o aumento do tempo em que paciente realizará a dialise. Esse aumento na sobrevida ocorreu apesar de os pacientes recentes serem mais idosos e foi prevalentemente mais pronunciada nos pacientes diabéticos. Os dados indicam que é fundamental a busca de melhora contínua de todos os itens do tratamento dialítico, beneficiando, assim, todos os pacientes submetidos a essa terapia.

Para Sousa et al. (2011), a infecção e a trombose compreendem as complicações mais comuns relacionadas ao uso do cateter. Essas complicações, além de graves, comprometem o estado clínico do paciente e prejudicam futuros acessos.

O fato de aplicar múltiplas punções no local da fístula aumenta o risco para infecções e hematomas, o que torna necessário uma constante vigilância, pois o acesso ideal e a preservação da rede venosa contribuem para um fluxo sanguíneo adequado para a realização da hemodiálise com baixo índice de complicações (Reinas, et al., 2012).

Segundo Neves Júnior et al. (2013), com o prolongamento da expectativa de vida dada a esses pacientes pelo próprio tratamento dialítico, o esgotamento do sistema venoso do membro superior pode levar à necessidade da confecção de FAVs de exceção. 
Research, Society and Development, v. 9, n. 11, e1609119715, 2020

(CC BY 4.0) | ISSN 2525-3409 | DOI: http://dx.doi.org/10.33448/rsd-v9i11.9715

Diante disso, percebe a necessidade de maior capacitação nesta área pelos profissionais de saúde, pois estão em contato direto com o paciente e necessitam observar as particularidades de cada um nos momentos críticos do tratamento, como os que envolvem a saúde mental, social e física, e desenvolver ações que propiciem melhoras do seu bem-estar (Pilger et al., 2010).

Nesse sentido, Silva et al. (2011), enfatiza a importância da atuação dos profissionais de modo mais próximo aos pacientes em tratamento hemodialitico, visando conhecer suas percepções frente as limitações enfrentadas, descobrir os possíveis comprometimentos decorrentes destas situações, bem como as adaptações necessárias em suas vidas para a concretização do tratamento.

\section{Conclusão}

A pesquisa possibilitou a consolidação de conhecimento sobre a doença renal crônica e a compreensão do perfil sociodemográfico e epidemiológico desses pacientes que constituise em sua maioria de indivíduos do sexo masculino, com idade compreendida entre 56 a 70 anos, estado civil casado, aposentado, residentes no estado do Piauí, com ensino fundamental incompleto e declarados católicos.

Quanto ao perfil epidemiológico, constatou-se como causa principal da lesão renal crônica, a hipertensão arterial sistêmica, seguida de diabetes mellitus. Observou-se ainda que a maioria dos entrevistados realizam diálise há um longo período de tempo e que o tipo de acesso vascular utilizado para a realização das diálises é fistula arteriovenosa. Em vista disso, nota-se que ao se conhecer o perfil dessa população, novas estratégias de cuidados em saúde podem ser traçadas para redução principalmente das taxas de morbidade e mortalidade.

Conclui-se, então, que a identificação e análise do perfil dos pacientes podem subsidiar os profissionais de saúde atuantes na área clínica, facilitando o manejo das atividades desenvolvidas em campo.

Assim, esse estudo atingiu o objetivo proposto, servindo como embasamento para reflexão dos atores envolvidos e aprimoramento do ensino acerca dessa temática, porém vale salientar que o estudo teve como limitações a aplicação do questionário em apenas uma instituição, não permitindo generalização dos dados, necessitando de novos estudos que contemplem o tema em questão, enfatizando aspectos clínicos e demográficos dos pacientes com doença renal crônica em tratamento hemodialítco. 


\section{Referências}

Almeida, M. I. C., Cardoso, M. S., Garcia, C. P. C., Oliveira, J. R. F. \& Gomes, M. L. F. (2013). Perfil dos pacientes renais crônicos de um hospital público da Bahia. Revista Enfermagem Contemporânea. 2(5)157-168. DOI: http://dx.doi.org/10.17267/23173378rec.v2i2.290

Alves, L. C., et al. (2007). A influência das doenças crônicas na capacidade funcional dos idosos do Município de São Paulo, Brasil. Cad. Saúde Pública, Rio de Janeiro,23(8)19241930.

Andrade, J. M. O., et al. (2014). Influência de fatores socioeconômicos na qualidade de vida de idosos hipertensos. Ciência \& Saúde Coletiva,19(8)3497-3504. DOI: https://doi.org/10.1590/1413-81232014198.19952013

Bastos, M. G., \& Kirsztajn, G. M. (2011). Doença renal crônica: importância do diagnóstico precoce, encaminhamento imediato e abordagem interdisciplinar estruturada para melhora do desfecho em pacientes ainda não submetidos à diálise. J Bras Nefrol. 33(1)93-108.

Bertolin, D. C., Pace, A. E., Kusumota, L., \& Haas V. (2011). Associação entre os modos de enfrentamento e as variáveis sociodemográficas de pessoas em hemodiálise crônica. Revista Escola de Enfermagem USP, 45(5)1070-1076. DOI: https://doi.org/10.1590/S008062342011000500006

Cordeiro, J. A. B. L., et al. (2009). Qualidade de vida e tratamento hemodialítico: avaliação do portador de insuficiência renal crônica. Rev. Eletr. Enf. 11(4)785-793.

Costa, F. G., Coutinho, M. P. L., Melo, J. R. F. \& Oliveira, M. X. (2014). Rastreamento da Depressão no Contexto da Insuficiência Renal Crônica. Temas em Psicologia, 22(2),445-455. DOI: http://dx.doi.org/10.9788/TP2014.2-14

Draczevski, L., \& Teixeira, M. L. (2011). Avaliação do perfil bioquímico e parâmetros hematológicos em pacientes submetidos a hemodiálise. Revista Saúde e Pesquisa, 4(1)15-22. 
Kirchner, R. M., Machado, R. F., Löbler, L., \& Stumm, E. M. F. (2011). Análise do estilo de vida de renais crônicos em hemodiálise. O mundo da saúde, 35(4)415-421.

Koche, J. C. (2011). Fundamentos de metodologia científica: teoria da ciência e iniciação à pesquisa. 1 ed. Petrópolis, RJ: Vozes.

Lopez, P. S., da Silva, D. P. C., Martin, L. C., \& Montovani, J. C. (2014). Could the type of treatment for chronic kidney disease affect the auditory system? Braz J Otorhinolaryngol. 80(1), 54-59. DOI: https://doi.org/10.5935/1808-8694.20140012

Madeiro, A. C., Machado, P. D. L. C., Bonfim, I. M., Braqueais, A. R., \& Lima, F. E. T. (2010). Adesão de portadores de insuficiência renal crônica ao tratamento de hemodiálise. Acta Paul Enferm. 23(4)546-551. DOI: http://dx.doi.org/10.1590/S0103-21002010000400016

Maldaner, C. R., Beuter, M., Brondani, C. M., Budó, M. D. L. D., \& Pauletto, M. R. (2008). Fatores que influenciam a adesão ao tratamento na doença crônica: o doente em terapia hemodialítica. Revista Gaucha de Enfermagem,29(4).

Melo, W. F., et al. (2014). Perfil epidemiológico de pacientes com insuficiência renal crônica: um estudo quantitativo. C\&D Revista Eletrônica da Fainor, 7(2)142-156.

Moura, L. D., Andrade, S. S. C. D. A., Malta, D. C., Pereira, C. A., \& Passos, J. E. F. (2015). Prevalência de autorrelato de diagnóstico médico de doença renal crônica no Brasil: pesquisa nacional de saúde, 2013. Revista Brasileira de Epidemiologia, 18(2)181- 191. DOI: https://doi.org/10.1590/1980-5497201500060016

Neves Junior, M. A. D., Petnys, A., Melo, R. C., \& Rabboni, E. (2013). Acesso vascular para hemodiálise: o que há de novo? Jornal Vascular Brasileiro, 12(3)221-225. DOI: http://dx.doi.org/10.1590/jvb.2013.044

Nunes, M. B., Santos, E. M., Leite, M. I., Costa, A. S., \& Guihem, D. B. (2014). Perfil epidemiológico de pacientes renais crônicos em programa dialítico. Revista de Enfermagem UFPE online, 8(1)69-76. 
Ortiz, A., Covic, A., Fliser, D., Fouque, D., Goldsmith, D., Kanbay, M., \& Wiecek, A. (2014). Epidemiology, contributors to, and clinical trials of mortality risk in chronic kidney failure. Lancet. 383(9931)1831-1843. DOI: https://doi.org/10.1016/S0140-6736(14)60384-6

Pereira, A. S., Shitsuka, D. M. Parreira, F. J., \& Shitsuka R. (2018). Metodologia da pesquisa científica 1. ed. Santa Maria, RS: UFSM, NTE.

Pilger, C., Rampari, E. M., Waidman, M. A. P., \& Carreira, L. (2010). Esc. Anna Nery, Rio de Janeiro, 14(4)677-683. DOI: https://doi.org/10.1590/S1414-81452010000400004

Pivatto, D. R., \& Abreu, I. S. (2010). Principais causas de hospitalização de pacientes em hemodiálise no município de Guarapuava, Paraná, Brasil. Rev Gaúcha de Enfermagem. 31(3)1-7. DOI: https://doi.org/10.1590/S1983-14472010000300015

Reinas, C. A., de Oliveira Nunes, G., \& de Mattos, M. (2012). O auto cuidado com a fístula arteriovenosa realizado pelos doentes renais crônicos da região sul de Mato Grosso. Revista Eletrônica Gestão \& Saúde. 3(1)505-519.

Silva, A. S. D., Silveira, R. S. D., Fernandes, G. F. M., Lunardi, V. L., \& Backes, V. M. S. (2011). Percepções e mudanças na qualidade de vida de pacientes submetidos à hemodiálise. Rev Bras Enferm, 64(5)839-844. DOI: https://doi.org/10.1590/S0034-71672011000500006

Silva, R. A. R., et al. Estratégias de enfrentamento utilizadas por pacientes renais crônicos em tratamento hemodialítico. Esc Anna Nery, 20(1), 147-154, 2016. DOI: https://doi.org/10.5935/1414-8145.20160020

Sousa, M. N. A., Sarmento, T. C., \& Alchieri, J. C. (2011). Estudo quantitativo sobre a qualidade de vida de pacientes hemodialíticos da Paraíba,Brasil. CES Psicología, 4(2)1-14.

Souza Júnior, E. Á., Trombini, D. D. S. V., Mendonça, A. R. D. A., \& Atzingen, A. C. V. (2015). Religião no tratamento da doença renal crônica: comparação entre médicos e pacientes. Revista bioética, 23(3)615-622. 
Valcanti, C. C., Chaves, É. D. C. L., Mesquita, A. C., Nogueira, D. A., \& Carvalho, E. C. D. (2012). Coping religioso/espiritual em pessoas com doença renal crônica em tratamento hemodialítico. Rev Esc Enferm USP, 46(4)838-845. DOI: https://doi.org/10.1590/S008062342012000400008

Vieira Júnior, J. M., \& Suassuna, J. H. R. (2013). O acometimento renal na hipertensão arterial e diabetes mellitus tipo 2: como identificar e prevenir - A visão do nefrologista. Revista Hospital Universitário Pedro Ernesto, 12. DOI: https://doi.org/10.12 957/rhupe.2013.7083

\section{Porcentagem de contribuição de cada autor no manuscrito}

Edildete Sene Pacheco - 20\%

Isabela Santana Macêdo - 20\%

Gabriela Santana Macêdo - 10\%

Aclênia Maria Nascimento Ribeiro - 10\%

Mariana Pereira Barbosa Silva - 5\%

Gabriela Oliveira Parentes da Costa $-5 \%$

Francinalda Pinheiro Santos - 5\%

Elton Filipe Pinheiro de Oliveira - 5\%

Felipe Nascimento Vidal - 5\%

Evellyn Stefanne Bastos Marques - 5\%

Agna Roberta Rodrigues de Sousa - 10\% 